\title{
Mid-Range, Coupling-Independent, Wireless Power Transfer with Parallel Resonators
}

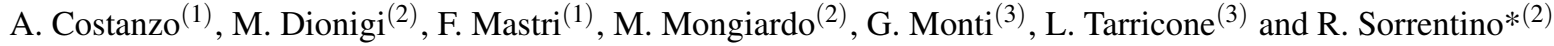 \\ (1) University of Bologna, Bologna, Italy \\ alessandra.costanzo@unibo.it, franco.mastri@unibo.it \\ (2) University of Perugia, Perugia, Italy \\ marco.dionigi@unipg.it,mauro.mongiardo@unipg.it, roberto.sorrentino@unipg.it \\ (3) University of Salento, Lecce, Italy \\ giuseppina.monti@unisalento.it, luciano.tarricone@unisalento.it
}

\begin{abstract}
This paper provides the design formulas for achieving a coupling-independent regime for a wireless power link consisting of magnetically coupled parallel resonators. It is demonstrated that for a strongly coupled link a performance independent of the coupling coefficient can be realized by an appropriate selection of the operating frequency.
\end{abstract}

\section{Introduction}

Near-field Wireless Power Transfer (WPT) is currently receiving considerable attention [1]-[3].

Among the possible implementations, a great attention has been paid to WPT links based on inductive coupling and considerable progress has been made in understanding the possibilities and limitations of such a kind of links [4]-[5]. In particular, in order to maximize the performance of the link, it has been demonstrated that it is convenient to use synchronous resonant schemes [4]. Usually, these schemes adopt a fixed-frequency regime and are realized by adding appropriate compensating capacitors which make the two coupled inductors resonating at the operating frequency of the link. In this regard, of particular interest are the analytical results presented in [5] demonstrating that, for a given link, the load can be selected in such a way to maximize either the power delivered to the load or the efficiency. This fixed-frequency regime has the advantage of providing the best performance; however, the optimal load is dependent on the coupling coefficient: a variation of the coupling coefficient imposes to modify the load to keep working with the best performance in terms of either power on the load or efficiency.

An attractive alternative to this fixed-frequency operating regime is the frequency-agile one that has been recently proposed in [6]. In more detail, referring to a WPT link using magnetically coupled series resonators, in [6] it has been shown that for a strongly coupled link a couplingindependent suboptimal performance can be realized by an appropriate selection of the operating frequency.

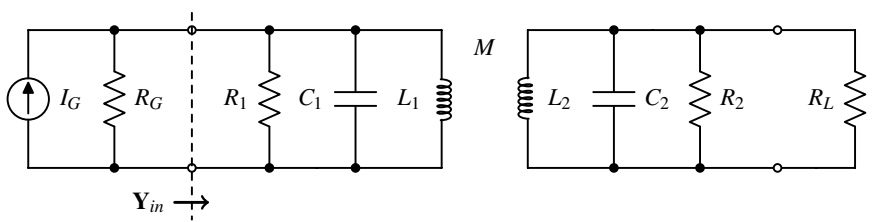

Figure 1. Schematic of the WPT link with parallel resonators and mutually coupled inductors.

In the present paper the theory developed in [6] is extended to the case of resonators using a parallel configuration. It is analytically demonstrated that when the coupling coefficient is greater than a threshold value, in addition to the main resonant frequency, two further frequencies exist for which the input admittance is purely resistive. It is also shown that, when the link is operated at these frequencies and the load has a given fixed value, the coupled parallel resonators act as a transformer and realize a constant, coupling-independent power transfer.

\section{Wireless Power Transfer Link with Parallel Resonators}

The WPT link analyzed in this paper is illustrated in Fig. 1. It is assumed that the resonators are synchronous, i.e. they have the same resonant angular frequency $\omega_{0}=$ $1 / \sqrt{L_{1} C_{1}}=1 / \sqrt{L_{2} C_{2}}$.

In order to generalize the circuit analysis for any possible operating frequency and power levels, it is convenient to make use of normalized quantities. Accordingly, the definition summarized in Table 1 and the normalized conductances reported in Table 2 are introduced.

\subsection{Main and secondary resonant frequen- cies}

Referring to Fig. 1, it is possible to calculate the expression of the input admittance $y_{i n}=\mathbf{Y}_{i n} / B_{0}$ and to verify that the input susceptance vanishes at $u=u_{0}=1 / \sqrt{\sigma}$, which will 
Table 1. Definitions

$\begin{array}{ll}\text { Normalized frequency } & u=\omega / \omega_{0} \\ \text { Susceptance slope parameter } & B_{0}=\sqrt{C_{1} / L_{1}} \\ \text { Transform ratio } & n=\sqrt{L_{1} / L_{2}} \\ \text { Coupling coefficient } & k=M / \sqrt{L_{1} L_{2}} \\ \text { Leakage coefficient } & \sigma=1-k^{2}\end{array}$

Table 2. Normalized conductances

$$
\begin{array}{ll}
g_{1}=1 /\left(B_{01} R_{1}\right)=1 / Q_{1} & g_{2}=1 /\left(n^{2} B_{01} R_{2}\right)=1 / Q_{2} \\
g_{G}=1 /\left(B_{01} R_{G}\right)=1 / Q_{G} & g_{L}=1 /\left(n^{2} B_{01} R_{L}\right)=1 / Q_{L} \\
g_{1 T}=g_{1}+g_{G}=1 / Q_{1 T} & g_{2 T}=g_{2}+g_{L}=1 / Q_{2 T}
\end{array}
$$

be denoted as the main resonant frequency. In addition, it can be demonstrated that the input admittance exhibits two further resonant frequencies (frequency bifurcation) when the following conditions are satisfied:

$$
\begin{gathered}
k^{2}>1-\frac{2}{g_{2 T}^{2}} \\
k \geq k_{b}=\sqrt{1-\frac{\left(1-\sqrt{1+2 g_{2 T}^{2}}\right)^{2}}{g_{2 T}^{4}} .} .
\end{gathered}
$$

The condition expressed in (1) is satisfied for all $k$ if $g_{2 T}<$ $\sqrt{2}$, as usually happens in practical cases. As per (2), it is satisfied for values of the coupling coefficient greater than a critical value $k_{b}$ denoted as the bifurcation coupling.

When (1) and (2) hold, the input susceptance also vanishes at the secondary resonant frequencies

$$
u_{ \pm}=\sqrt{\frac{2-\sigma g_{2 T}^{2} \pm \sqrt{\left(2-\sigma g_{2 T}^{2}\right)^{2}-4 \sigma}}{2 \sigma}}
$$

\subsection{System behavior at the secondary reso- nant frequencies}

The system behavior at the main resonance is well known and it is not discussed here. In this paper, the analysis focuses on the secondary resonances with the aim of demonstrating that it is possible to achieve a couplingindependent operating regime provided that the conditions expressed in (1)-(2) are satisfied .

At $u=u_{ \pm}$the normalized input conductance, for all values of $k$ is

$$
g_{\text {in }}=g_{1}+g_{2 T}
$$

which corresponds to the unnormalized input conductance

$$
G_{i n}=G_{1}+\frac{1}{n^{2}}\left(G_{2}+G_{L}\right)
$$

This shows that, from the input side, the coupled resonators act as an ideal transformer with transform ratio equal to $n$ and independent of $k$. In these conditions the normalized output power, $p_{L}$, and the efficiency, $\eta=p_{L} / p_{G}$ are also independent of $k$

$$
\begin{gathered}
p_{L}=\frac{g_{L}}{\left(g_{1 T}+g_{2 T}\right)^{2}} \\
\eta=\frac{g_{L}}{g_{1 T}+g_{2 T}} .
\end{gathered}
$$

\section{Conclusion}

In this paper a WPT link using magnetically coupled parallel resonators has been analyzed and the conditions for achieving a coupling-independent operating regime have been derived.

It has been demonstrated that, when the magnetic coupling coefficient is greater than a critical value, in addition to the main resonance, two secondary resonant frequencies exist where the input admittance of the link is purely resistive.

It is also shown that a coupling-independent performance can be realized by adopting a frequency-agile scheme where one of the two secondary resonant frequencies is selected as the operating frequency of the link. In this way, constant output power and efficiency can be obtained, regardless of coupling coefficient variations (due to e.g. distance variations or coil misalignment). Analytical results are provided making use of normalized quantities, so that they can be scaled to accomplish different WPT applications, in terms of power levels and frequency range.

\section{References}

[1] M. Fu, T. Zhang, C. Ma, and X. Zhu, "Efficiency and Optimal Loads Analysis for Multiple-Receiver Wireless Power Transfer Systems," IEEE Transactions on Microwave Theory and Techniques, vol. 63, no. 3, pp. 801-812, Mar 2015.

[2] C. Florian, F. Mastri, R. Paganelli, D. Masotti, and A. Costanzo, "Theoretical and numerical design of a wireless power transmission link with GaN-based transmitter and adaptive receiver," IEEE Transactions on Microwave Theory and Techniques, vol. 62, no. 4, pp. 931-946, April 2014.

[3] A. Costanzo, M. Dionigi, M. Masotti, M. Mongiardo, G. Monti, L. Tarricone, and R. Sorrentino, "Electromagnetic energy harvesting and wireless power transmission: A unified approach," Proceedings of the IEEE, vol. 102, no. 11, pp. 1692-1711, Nov 2014.

[4] A. Kurs, A. Karalis, R. Moffatt, J. D. Joannopoulos, P. Fisher, and M. Soljacic, "Wireless power transfer via strongly coupled magnetic resonances," Science, vol. 317, no. 5834, pp. 83-86, 2007.

[5] M. Dionigi, M. Mongiardo, and R. Perfetti, "Rigorous network and full-wave electromagnetic modeling of wireless power transfer links," IEEE Transactions on Microwave Theory and Techniques, vol. 63, no. 1, pp. 65-75, Jan 2015. 
[6] F. Mastri, A. Costanzo, and M. Mongiardo, "CouplingIndependent Wireless Power Transfer," IEEE Microwave and Wireless Components Letters, vol. 63, no. 3, pp. 222-224, Feb 2016. 